\title{
A Semantic Reasoning Method Towards Ontological Model for Automated Learning Analysis*
}

\author{
Kingsley Okoye $\mathrm{e}^{\mathrm{a}^{*}}$, Abdel-Rahman H. Tawil ${ }^{\mathrm{a}}$, Usman Naeem ${ }^{\mathrm{a}}$ and Elyes Lamine ${ }^{\mathrm{b}}$

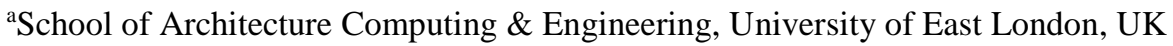 \\ \{u0926644, A.R.Tawil, U.Naeem\}@uel.ac.uk \\ bUniversité de Toulouse, Mines-Albi, CGI, Campus Jarlard, Albi Cedex 09, France \\ Elyes.Lamine@mines-albi.fr
}

\begin{abstract}
Semantic reasoning can help solve the problem of regulating the evolving and static measures of knowledge at theoretical and technological levels. The technique has been proven to enhance the capability of process models by making inferences, retaining and applying what they have learned as well as discovery of new processes. The work in this paper propose a semantic rule-based approach directed towards discovering learners interaction patterns within a learning knowledge base, and then respond by making decision based on adaptive rules centred on captured user profiles. The method applies semantic rules and description logic queries to build ontology model capable of automatically computing the various learning activities within a Learning Knowledge-Base, and to check the consistency of learning object/data types. The approach is grounded on inductive and deductive logic descriptions that allows the use of a Reasoner to check that all definitions within the learning model are consistent and can also recognise which concepts that fit within each defined class. Inductive reasoning is practically applied in order to discover sets of inferred learner categories, while deductive approach is used to prove and enhance the discovered rules and logic expressions. Thus, this work applies effective reasoning methods to make inferences over a Learning Process Knowledge-Base that leads to automated discovery of learning patterns/behaviour.
\end{abstract}

Keywords: Process Model, Learning Process, Ontology, Semantic Reasoning

\section{Introduction}

Ontology is one of the scientifically proven technique that is used to model different kinds and structure of objects, events and processes as they happen in reality. The concept can be layered on top of existing information asset to provide a more conceptual analysis of real time processes capable of providing real world answers that are closer to human understanding [1]. Ontology presents to the data science world the capability of using semantics to classify instances to explain the dependent variables in terms of independent ones; which is a great way to compliment the way we look at processes. The concept of semantic annotations and reasoning makes it possible to match same ideas as well as use the coherence and structure itself to inform and answer questions about relationships learning objects share within information knowledge base. The various units/activities within a learning process model can be related to exactly one case 
and assigned a case identifier [2] which results in automatic creation of workflow processes [3] and can help to maintain the resulting hierarchy correctly. This approach is made possible by using the semantic annotation scheme to represent sets of various entities, properties and classes within the learning knowledge base and create inferences capable of providing new knowledge or a richer set of intelligence within the model.

In this paper, we use ontology web rule languages and schema to discover sets of relationships that can be found within a learning process knowledge base. As a result, suitable learning paths are determined by means of semantic reasoning, which is then used to address the problem of extracting useful patterns from captured data source to provision of knowledge.

The rest of the paper is structured as follows; in Section 2, we present a description of learning process model and how we apply the representations for learning activities to draw conclusions and make predictions based on analysis of captured learning data. Section 3 shows the proposed semantic process model, describing in detail its ontological representation and reasoning using Ontology Web languages. The prototype implementation and preliminary outcomes was presented in Section 4, and in Section 5, we analyse and discuss appropriate related work in this area of study. Finally, Section 6 concludes the paper and points out directions for future research.

\section{Ontological Description of Process Model}

Ontological description of process model is based on computer logic programming [4], and has been related to the natural process of human thinking. Lumpe [5] mentions that Inductive intelligent is the process of reasoning from the particular to the general which involves the observation of particular events or data. The approach associates new content with prior knowledge; which can lead to unrelated data being discovered, examined and further grouped and labelled in order to draw conclusions or make predictions based on the analysis of the data. Following the works in [6] and [7], the ability to analyse information and create concepts is fundamental to ontological reasoning and can be applied towards automation of learning processes, as we define below;

Step I: Examine the Learning Knowledge base to define unrelated entities.

Step 2: Group entities with common attributes and provide descriptive labels to objects or data property.

Step 3: Identify relationships in order to generalise, predict and extract patterns from the existing properties.

Step 4: Apply discovered patterns to a new and different context to demonstrate understanding.

Step 5: Check that all entities within the discovered classes are true and at least falls within the universal restriction of validity by definition, and that there are no inconsistency of data or repeatable contradicting discovery.

The purpose of the definition is to use the concept of ontological model to describe and understand learning process reality based on captured knowledge or historic data, and the ability to provide a link between learning objects or data types. 


\section{Concept Matching and Association of Variables}

Association Rule Learning aims at finding rules that can be used to predict the value of some response variables that has been identified as being important just like decision systems [8] but without focusing on a particular response variable. The rule aims at creating rules of the form:

\section{IF X THEN Y}

where $\mathbf{X}$ is often called the antecedent and $\mathrm{Y}$ the consequent. Thus, $\mathrm{X} \Rightarrow \mathrm{Y}$

This rule is similar and can be related to the Semantic Web Rule Language, SWRL [9] which is useful especially to provide more ontological description and enhancement to learning process model.

The SWRL rule has the form; $a t a m^{\wedge}$ atam (antecedent).... $\rightarrow$ atam ${ }^{\wedge}$ atam (consequent).

Association rule learning strongly supports the use of metrics frequently expressed in the form of support and confidence. These expressions help in measurement of the strength of the association between learning objects. Support determines how often a rule is applicable to a given data set which means the fraction of instances for which both antecedent and consequent hold. Thus, a rule with high support is more useful than a rule with low support. A rule that has low support may occur simply by chance and is likely to be irrelevant from a learning perspective because it may not be profitable to monitor, recommend and promote learning activities or learning patterns.

Support is used to evaluate learning process models and its execution, where:

$\mathrm{Nx}$ is the number of instance for which, $\mathrm{x}$, learning activity holds.

Ny the number of instances for which learning activity y holds.

And $\mathbf{N} \mathbf{x}_{n} \mathbf{y}$ is the number of instances for which activity $\mathbf{x}$ and $\mathbf{y}$ holds.

Consequently, support for the rule $X \Rightarrow Y$ is described as

Support, $s(X \Rightarrow Y)=N x_{y} y / N \quad:$ where $N$ is the total number of instances.

Confidence, on the other hand, measures the reliability of the inference made by a rule over a learning process. Thus, for a give rule of the form $X \Rightarrow Y$, the higher the confidence, the more likely it is for the consequent $Y$ (learning pattern extension) to be represented within the learning process that contains $X$ (learning patterns). Confidence measures the conditional probability that the extension $Y$ will happen given $X$.

\section{Confidence, $c(X \Rightarrow Y)=N x_{n} y / N x$}

Overall, inferences made by an association rule learning suggest co-occurrence of relationships between items in the antecedent $(X)$ and consequent $(Y)$ of the rule. Therefore, for every given set of activities or item set, there exist rules having support $\geq$ $\operatorname{minSup}$, and confidence $\geq \operatorname{minConf}$, where: $\operatorname{minSup}$ and $\operatorname{minConf}$ are respectively the corresponding support and confidence thresholds. 
In Learning Process models, these metrics can be used to dramatically reduce the exploration or drilling down space when constructing the set of frequent activity logs. The simple requisite is that $X$ and $Y$ are non-empty and any variable appears at most once in $\mathrm{X}$ or $\mathrm{Y}$. For instance,

$$
\text { IF Learner(X) AND hasLearning_Activities THEN hasPartLearning_Pracess(Y) }
$$

Thus, Learner(?X), hasLearning_Activities(?X, Activity) -> hasPartLearning_Process(?X, ?Y)

This approach has been used to provide process specification and expressive language formats that are logically and fundamental to knowledge representation such as the Knowledge Interchange Format (KIF)[10] which makes it possible to understand the meaning of logic expressions through Declarative Semantics. For instance, it can be expressed that "Еvery Learner has a Learning_Activity". Thus;

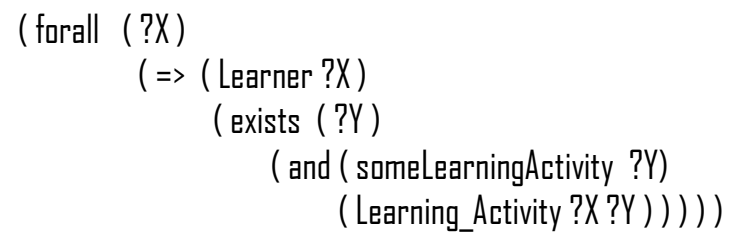

Consequently, Every Learning_Activity is part of a Learning_Process and must have same kind of a Learner. Thus the expression;

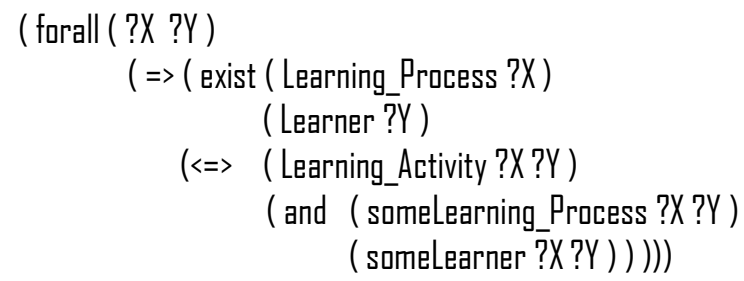

Such rule expressions suggest that a strong relationship exist between the Learning_Process and the Learner. This is because Learner $(X)$ has_Activities described as a Learning_Activity, and Learning_Activity has been described as PartDfLearning_Process.

Designers of knowledge base systems can use this type of rule expressions to help identify new opportunities especially for enhancement of process models. Association rule is now recently being used in application domains such as the web mining and scientific data analysis. The association patterns reveals interesting connection among domain entities, the individual classes and object/data types to provide a better understanding of how the different elements within the Learning Process Knowledge base relate and interact with each other. In the next section, we describe and implement the concept of ontological reasoning of learning activities capable of deducing inferences based on such design rule-base semantic approach to automated learning. The focus is 
on implementing the learning objects/property restrictions to define the classes and relationships of the entities within the Learning Knowledge-base.

\subsection{Ontology Model for Automation of Learning and Reasoning}

Based on ontological vocabularies, inductive reasoning has as input data types from which a possible sameValuesfram or believable generalisation is computed. This technique is an existential restriction, which describes the set of individuals that have at least one specific kind of relationship to individuals that are member of a specific class. It is a relationship that exist between two individuals, i.e concept assertion that hold between two objects. On the other hand, deductive reasoning which has been generally adopted in the Semantic Web context assumes an al/Valuesfram restriction, whereby given a set of general axioms, precise and definite conclusions are drawn by the use of a formal proof. This technique is referred to as universal restriction, which describes the set of individuals that for a given property only have relationships to other individuals that are members of a specific class.

Fig. 1 is an Ontology Web Language (OWL) version 2 model of our proposed Learning Ontology, implemented in Protégé 4.3 and reasoned upon using Pellet 2. Protégé 4.3 OWL editor [11] supports Description Logic (DL) Queries [12] and SWRL rules [9].

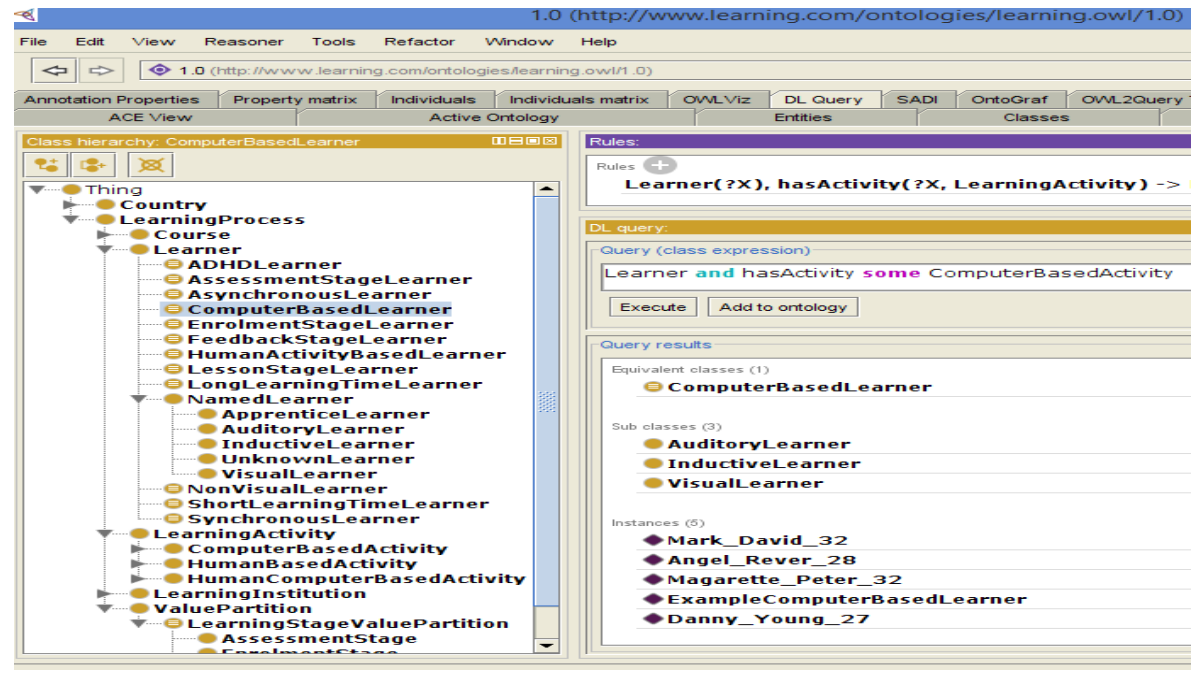

Fig 1. Learning Model in Protégé 4.3 Editor with SWRL Rule and DL Query

In Fig. 1, we use the protégé Editor to construct ontology that expresses the functionality of the Learning model in terms of individual learning characteristics. The Cases within the model were defined as sub-class of the main class LearningProcess. The class expression is based on the OWL syntax primarily focused on collecting all information about a particular class or individual into a single construct, called a Frame. The DL Query provides the platform for searching the classified ontology to infer the learning activities of any named individual. The result of the logic expression and reasoning is what we use to show the process model and automated discovery of learning patterns. 
The tactics aims at discovering rules similar to the Association Rule [13] as previously described in section 3.0, but then without focusing on a particular variable to discover user interaction patterns and then respond by making decisions based on adaptive rules centred on the captured user profiles. The goal is to discover and create rules of the same form;

$$
\mathbf{X} \Rightarrow \mathbf{Y}(I F \mathbf{X} T H E N \mathbf{Y})
$$

where $\mathbf{X}=$ Learning pattern (Antecedent) and $\mathbf{Y}=$ Learning pattern extension (Consequent)

e.g. Learner (?X) , hasActivity (?X, ?LearningActivity) -> hasLearningProcess (?X, ?Y)

Learner (?X) , hasLearningProcess (?X, ?Y), hasLearningActivity (?ComputerBasedActivity, ?Z) -> hasComputerBasedLearner (?X, ?Z)

Driven by the variables as defined in the Learning Ontology in Fig. 1, and the OWL 2 XML file Fig. 2, the resulting rules expressions Fig. 3, were derived to improve the reasoning capability and semantics of the learning process model.

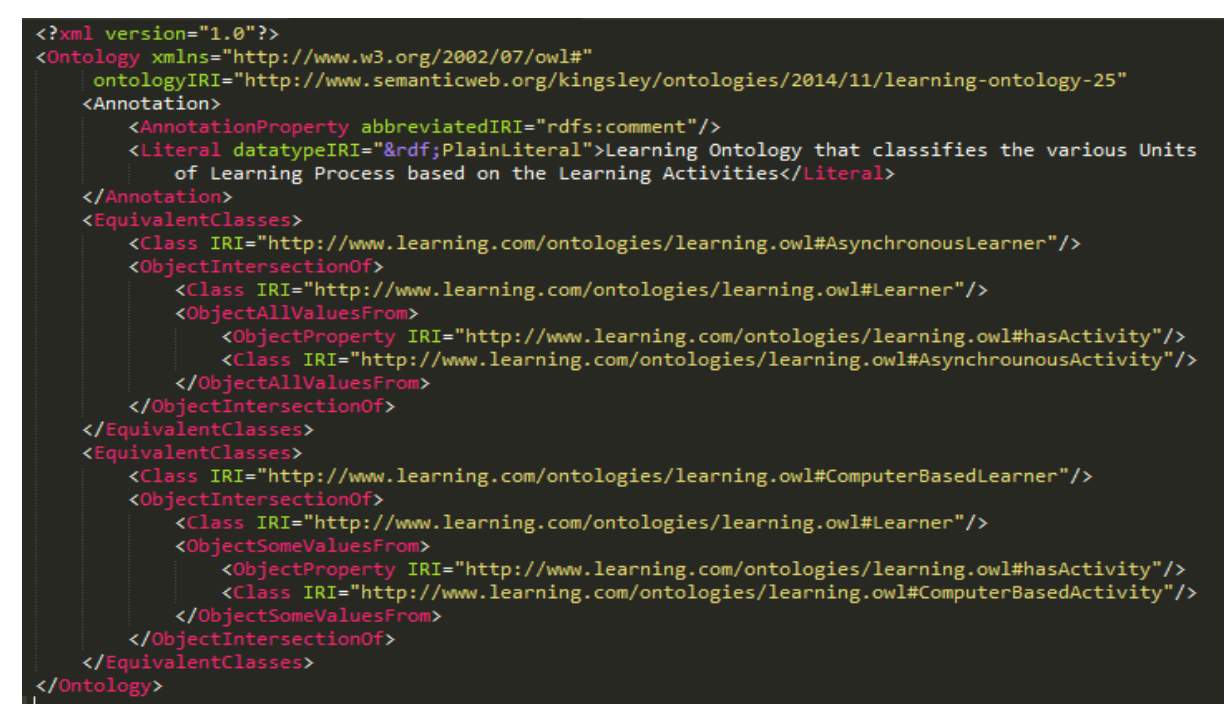

Fig [2]. A fragment of the Learning Ontology OWL 2 XML file in Protégé

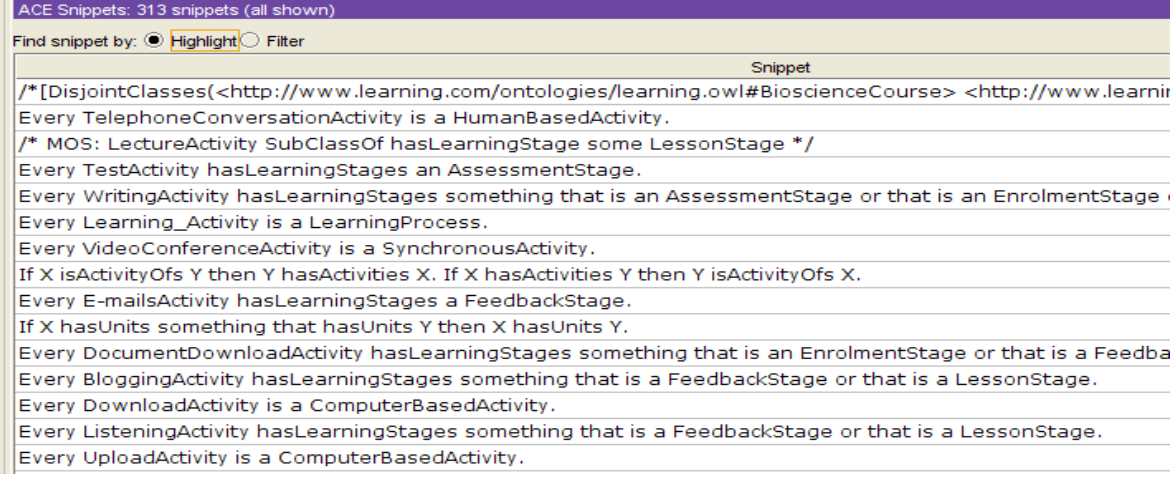

Fig [3] Fraction of rules executed in the Learning Ontology 


\section{Discussion}

Automation of learning process involves the flow of activities within a learning knowledge-base technically described as Workflow. Being able to use the Reasoner to automatically compute the class hierarchy of the activities within the knowledge-base is one of the major benefits of building ontology using OWL, SWRL Rules and DL Query. Annotation properties are used to add information (Metadata - data about data) to the classes, individuals and object/data properties in the ontology. The proposed Learning Model Ontology allows the meaning of properties to be enhanced through the use of property characteristics and classification of discoverable entities. We utilize the main function offered by the Reasoner to help in checking for consistency in the model; to test whether or not a class is a subclass of another class, or checking whether or not it is possible for a class to have any instances. This means a class is said to be inconsistent if it does not have any instances.

By performing such test (i.e. Classification) it becomes possible to compute the inferred activity hierarchy. The functional property assertion allows this condition, where: for a given class, there can be at least one individual that is related to the class by means of the restriction property. In OWL, a Restriction describes class of individuals based on the relationship the members of the class participate in. In the proposed model, we describe the class Learner to be a subclass of the LearningProcess. The necessary condition is: if something is a Learner, it is necessary for it to be a participant of the class LearningProcess and necessary for it to have a kind of sufficiently defined condition and relationship with other classes e.g. LearningActivity, Learninglnstitution, Course, LearningStageValuePartition etc. From the example in Fig. 1, we show that a ComputerBasedLeагпег is a subclass of, amongst other NamedLearners, a Learner and also a subclass of the LearningActivity class that have at least one Activity that is ComputerBased. This assertion is achieved through the Restriction Property. The property is used to infer anonymous classes (Unnamed classes) that contains all of the individuals that satisfies the restriction, in essence, all of the individuals that have the relationship required to be a member of the class. In effect, the necessary and sufficient Condition makes it possible to implement and check for consistency in the model which means that it is necessary to fulfil the condition of Object/Data Property Restriction - for any individual to become a member of a class.

\section{Related Works}

Learning patterns or behaviours can be discovered as a consequence or condition of a Rule. d'Amato et al [14] notes that various methods have been proposed in literature which are directed towards obtaining a more expressive model from knowledge bases [15]. The authors [14] argue that classification is a fundamental task for a lot of intelligent applications, and that classifying through logic reasoning may be both too demanding and frail because of inherent incompleteness and complexity in the knowledge bases. However, they observe that these methods adopt the availability of an initial drawing of ontology that can be automatically enhanced by adding or refining concepts, and have been shown to effectively solve learning modelling problems using Description Logics particularly those based on classification, clustering and ranking of individuals. 
Learning Process modelling has been tackled over the years by customising Machine Learning methods such as Instance Based Learning [16] and Support Vector Machine (SVM) [17] to Description Logics (DLs) [12] queries which is the standard theoretical foundation upon which semantic web languages such as OWL and SWRL are based.

According to [18] and [19] Bayesian models have paved way for new machinelearning algorithms with more powerful and more human-like capabilities. Semantic web ontology and its application cannot be explained without mentioning the Bayesian theory of probability [20][21]. The Bayesian probabilistic theory have been proven to be one of the few mathematical interpretation of predictive concepts for representing a state of knowledge, thus, an extension of logic proposals that enables reasoning with hypothesis whose true or false values is uncertain. Bayesian model is based on 3 vital probes: What are the content of probabilistic theories? How can they be used to support reasoning? And how can they themselves be reasoned upon? The hypotheses are measured by computing the Bayes' rule, where: Probability, $P(x \backslash h, T)$ measures how well each argument predicts the data and the initial marking or likelihood. $P(h \backslash T)$ expresses the plausibility of the hypotheses given the users background knowledge. The posterior probability, $P(h \backslash x, T)$, is proportional to the result of the two expressions representing the level of certainty in each of the hypothesis given both the constraints of the background theory $\mathrm{T}$, and observed data $\mathrm{x}$.

According to Tenenbaum et al [22], the challenge comes in specifying hypotheses and probability distributions that support Bayesian inference for a given task/domain. The authors argue that both structured knowledge and statistical inference are necessary to explain the nature, use and acquisition of such human knowledge and further introduced a theory-based Bayesian framework for modelling inductive learning and reasoning. Explicitly, the problems of modelling learning processes can be solved by transforming ontology population problem to a classification problem where, for each entity within the ontology, the concepts (classes) to which the entities belongs to have to be determined (i.e, classified) [14][1]. Generally, these approaches assume that there already exist a probabilistic and/or fuzzy knowledge-base upon which this methods are able to predict the patterns/behaviour (hence, the classification) of new but not previously observed object/data types within the process.

Inductive and deductive reasoning methods can be used as building block towards the development of probabilistic learning knowledge-bases, by learning the probability that an inclusion axiom or concept assertion holds between two objects. The authors in [14] argue that in presence of noisy and inconsistent knowledge-bases that could be highly probable in a distributed environment such as the world wide web, that deductive reasoning is no more applicable since it requires correct/true premises; whereby if all premises are true and the rules of the deductive logic are followed, then the conclusion reached is necessarily true. On the other hand, inductive reasoning which is grounded on the generalisation of specific instances/assertions rather than correct premises, allows the formulation of conclusions even when inconsistent or noisy knowledge bases are being considered. Unlike deductive reasoning, inductive reasoning allows for the possibility that the conclusion is false, even if all of the premises are true and does not 
rely on universal restrictions over a closed axiom to draw conclusions. Currently, inductive reasoning is the main practice for logical reasoning, obtaining conclusions that are believed by the scientific community to be the most probable explanation of observed phenomena.

Reasoning on ontological knowledge plays an important role in the semantic representation of processes such as learning process. This is possible because semantic reasoning allows the extraction and conversion of explicit information into some implicit information, for instance, the intersection or union of classes, description of relationships and concepts/role assertions. Thom et al [23] describes Workflow Activity Patterns (WAPS) as common structures involving the interaction between individual entities and the control-flow constructs used to model the semantics of activities as they are being performed. Workflow systems assume that a process can be divided into small, unitary actions, called Activities [3]. To perform a given process, one must perform the set (or perhaps a subset) of the activities that comprise it. Hence, an Activity is an Action that is a semantic unit at some level, which can be thought of as a function that modifies the state of the process in terms of the semantics of the patterns and can be discovered automatically by means of semantic reasoning [1].

\section{Conclusion and Future Work}

The work in this paper uses ontological schema/vocabularies to describe and propose a semantic rule-based approach that supports automated computing of different patterns within a learning process knowledge-Base through Semantic Reasoning. The method is proposed in order to address the problem of determining the presence of different learning patterns in process models. Any pattern or learning behaviour can be discovered as a consequence or condition of a Rule. Ontology provides us with benefits in discovery, flexible access and information integration due to the inherent connectedness (inference), concept matching and reasoning capability. This characteristic is the ability to match same idea as well as use the coherence and structure itself to inform and answer questions about relationships the learning objects (process instances) share amongst themselves within the Learning model.

Future work will focus on applying process mining techniques to provide better support for automated learning systems by means of semantic reasoning. The goal is to cover the whole spectrum of the approach presented in this paper; to help improve engagements within learning execution environments by adopting the main tools offered through conventional process mining for analysing process event data logs.

\section{References}

1. Okoye K., Tawil A.R.H., Naeem U., Bashroush R., Lamine E.: A Semantic Rule-Based Approach Supported by Process Mining for Personalised Adaptive Learning. In: Procedia Computer Science, Vol. 37(c), pp. 203-210. (2014)

2. Van der Aalst W.M.P.: Process Mining: Discovery, Conformance and Enhancement of Business Processes. Springer (2011) 
3. Ferreira D.L, Thom L.H.: A Semantic Approach to the Discovery of Workflow Activity Patterns in Event Logs. Journal of Business Process Integration and Management, Inderscience Publisher, Vol. 6(1) (2012)

4. Nguyen, T.H., Vo, B.Q., Lumpe, M., Grundy, J.: KBRE: a framework for knowledge-based requirements engineering. Journal of Software Quality. Volume 22, Issue 1, pp 87-119, March (2014)

5. Lumpe, A.: Forms of Models and Induction. Seattle, WA: Seattle Pacific Uni. Lecture notes (2007)

6. Jepbarova, N.: Survey of Instructional Strategies. Lecture notes EDU 6526, March 7 (2007)

7. Barbieri, D., Braga, D., Ceri, S., Valle, E.D., Yi Huang, Tresp, V., Rettinger, A., Wermser, H.: Deductive and Inductive Stream Reasoning for Semantic Social Media Analytics. Intelligent Systems, IEEE, vol.25, no.6, pp.32,41, Nov.-Dec. (2010)

8. Yarandi, M., Jahankhani, H.,Tawil, A.R.H.: Towards Adaptive E-Learning using Decision Support Systems. International Journal of Emerging Technologies in Learning. Vol 8. pp. $44-51$ (2013)

9. SWRL: A Semantic Web Rule Lang "http://www.w3.org/Submission/2004/SUBM-SWRL20040521/"

10. Knowledge Interchange Format. http://cl.tamu.edu/discuss/kif-100101.pdf Accessed [Dec. 2014]

11. Protégé Editor. http://protege.cim3.net Accessed [June $4^{\text {th }}$ 2014]

12. Baader, F., Calvanese, D., McGuinness, D.L., Nardi, D., Patel-Schneider, P.F.: editors. Description Logic Handbook. Cambridge University Press (2003)

13. Han, J., Kamber, M., Pei, J.: Data Mining: Concepts and Techniques. The Morgan Kaufmann Series in Data Management Systems, [ $3^{\text {rd }}$ Ed] Morgan Kaufmann Publishers, July (2011)

14. d'Amato, C., Fanizzi, N., Esposito, F.: Query answering and ontology population: An inductive approach. In Bechhofer, S., Hauswirth, M., Hoffmann, J., Koubarakis, M., eds.: Proc. of the 5th Euro. Semantic Web Conf., ESWC2008. Vol. 5021 of LNCS, Spr 288-302 (2008)

15. Lehmann, J., Hitzler, P.: Concept learning in description logics using refinement operators. $M a$ chine Learning, 78:203-250 (2010)

16. Mitchell, T.: editor. Machine Learning. McGraw Hill (1997)

17. Shawe-Taylor, J., Cristianini, N.: editors. Support Vector Machines \& other kernel-based learning methods. Cambridge Uni. Press (2000)

18. Kemp, C. et al.: Semi-supervised learning with trees. Advances in Neural Info. Processing Syst. Vol.(16), pp. 257-264, MIT Press (2004)

19. Griffiths, T.L., Chater, N., Kemp, C., Perfors, A., Tenenbaum, J.B.: Probabilistic models of cognition: exploring representations \& inductive biases. In: Trends in Cognitive Sciences, Vol. 14(8) pp. 357-364 (2010)

20. McGrayne, S.B.: The Theory That Would Not Die, p. 10., at Google Books (2011)

21. Fienberg, S.E.: When did Bayesian Inference become "Bayesian"? Bayesian Analysis, vol.1 (1), pp. 1-40 (2006)

22. Tenenbaum, J.B., Griffiths, T.L., Kemp, C.: Theory-based Bayesian models of inductive learning and reasoning. In: TRENDS in Cognitive Sciences Vol.10 No.7 July (2006)

23. Thom L.H., Reichert, M., Iochpe, C.: Activity patterns in process-aware information systems: basic concepts and empirical evidence. J. of Business Process Integration \& Mangt, Vol. 4(2), pp. 93$110(2009)$ 